\title{
Nano-loaded natural killer cells as carriers of indocyanine green for synergetic cancer immunotherapy and phototherapy
}

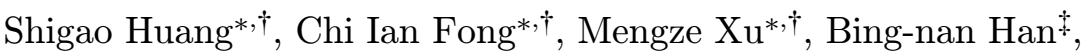 \\ Zhen Yuan*,+, and Qi Zhao*,ं, \\ *Cancer Center, Faculty of Health Sciences, University of Macau \\ Taipa, Macao SAR, P. R. China \\ $\dagger$ Institute of Translational Medicine \\ Faculty of Health Sciences, University of Macau \\ Taipa, Macao SAR, P. R. China \\ *School of Life Science \\ Zhejiang Sci-Tech University \\ Zhejiang, P. R. China \\ \$zhenyuan@um.edu.mo \\ 『qizhao@um.edu.mo; zhaoqi@alumni.cuhk.net
}

Received 29 January 2019

Accepted 20 March 2019

Published 3 April 2019

\begin{abstract}
To improve the efficacy of traditional chemotherapy and radiotherapy and reduce their serious side effects, further efforts need to be exerted to identify better cancer therapeutic options that are effective, affordable, and acceptable to patients. In this study, a novel theranostic agent was produced to perform synergetic cancer immunotherapy and phototherapy. The theranostic agent, named natural killer (NK) cells carrying indocyanine green loaded liposomes was synthesized NK cells with ICG nanoparticles to serve as the agent for a newly-established cancer treatment. It is expected that the developed synergistic therapy can pave a new avenue for improved efficacy of cancer theranostics.
\end{abstract}

Keywords: Nanoparticle; NK cell; photothermal therapy; cancer theranostics.

\section{Introduction}

To date, a number of therapy approaches have been developed in oncology, which include small molecules target therapy, ${ }^{1,2}$ antibody drug therapy, ${ }^{2-12}$ nanoparticle-based therapy, ${ }^{13-15}$ and immunotherapy. ${ }^{16}$ More importantly, immunotherapy that boosts the body's own immune system to kill cancer cells was established as a category of cancer

$\S$, Corresponding authors.

This is an Open Access article published by World Scientific Publishing Company. It is distributed under the terms of the Creative Commons Attribution 4.0 (CC-BY) License. Further distribution of this work is permitted, provided the original work is properly cited. 
treatment in the late 19 th century. ${ }^{17}$ Meanwhile, natural killer (NK) cells, as an essential part of the immune system, have the capability to spontaneously kill abnormal cells without prior sensitization. Different from T cells, NK cells that are routinely used in preclinical tests and clinical practice as part of the innate immune system can offer a fast immune response in recognizing and defending against the cancer cells. ${ }^{18-20}$ In particular, NK92 cells derived from NK lymphoma are considered to have high cytotoxicity over malignant cells, to be relatively long-lived when expanded ex vivo, and to be safe and more frequently used in clinical studies. ${ }^{21}$ In this study, an NK92-derived and highly toxic interleukin-2-independent cell line, ${ }^{22}$ NK-92MI cells were used in order to recognize and target the cancer cell antigens along cellular surface for cancer therapy. ${ }^{23} \mathrm{NK}-92 \mathrm{MI}$ cells can secrete perforin and granzymes that are normally stored inside the secretory lysosomes. ${ }^{24}$

In addition, photothermal therapy (PTT) is a robust tool for noninvasive cancer treatment owing to its high selectivity, low systemic toxicity, and negligible drug resistance. PTT makes full use of photothermal agents with strong optical absorption in the near-infrared (NIR) region, which can be activated by using NIR light to kill cancer cells. ${ }^{25-27}$ Interestingly, indocyanine green (ICG) that is the only contrast agent approved by the United State Food and Drug Administration (U.S. FDA) for clinical applications, has be adopted as a photothermal agent for cancer theranostics due to its attractive light absorption property in the NIR window $(700 \mathrm{~nm}-1000 \mathrm{~nm}) .{ }^{28}$

However, a potential concern on ICG is that it has poor aqueous stability, rapid degradation and fast clearance by the body, which significantly reduces the efficacy of $\mathrm{PTT}{ }^{29}$ Therefore, it is advisable to encapsulate ICG in a drug delivery system in order to resolve the basic limitations and maximize its biological applications. ${ }^{30}$ To date, various nanocarriers have been developed to encapsulate ICG to improve the efficacy of cancer treatment. For example, Kirchherr et al. demonstrated that encapsulated ICG within the Solutol HS 15 micelles exhibited lower aqueous aggregation and improved aqueous stability for over four weeks as compared to that without encapsulation. ${ }^{31}$

In this study, ICG-loaded nanoparticles (NPs) are synthesized, in which liposomal ICG nanoparticle can also serve as photothermal agents for

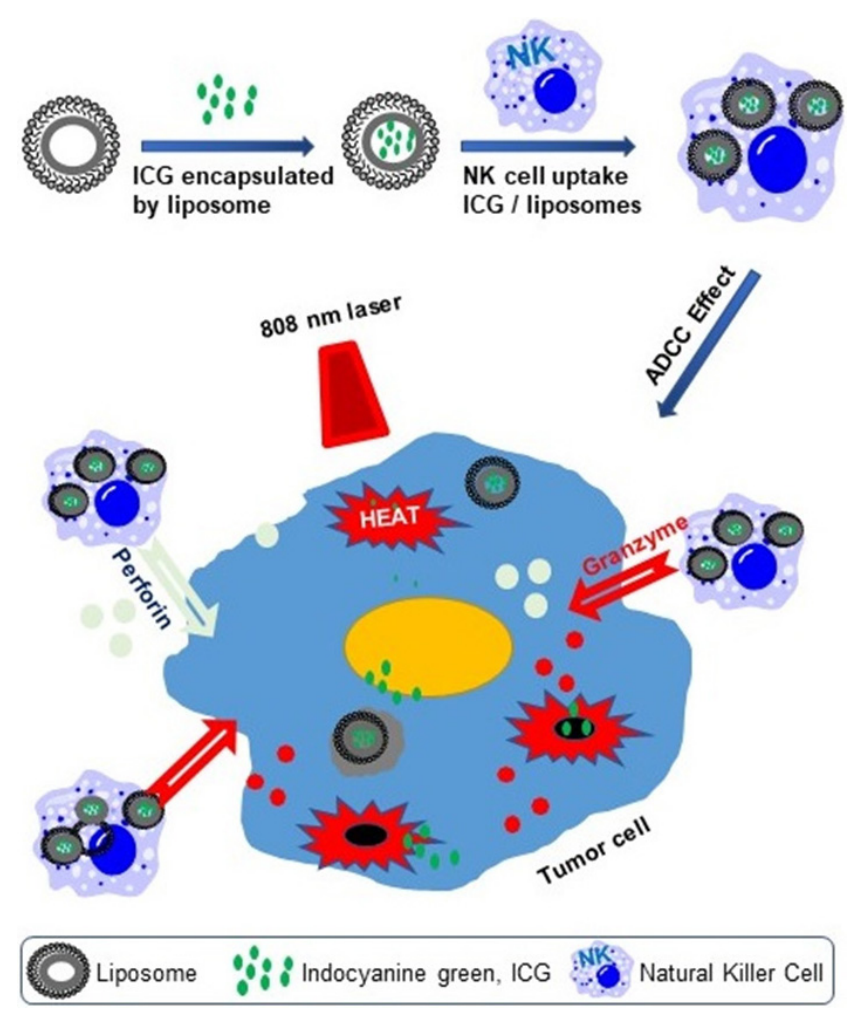

Fig. 1. Schematic of natural killer cell (NK) carrying indocyanine green (ICG) loaded liposomes to conduct both immunotherapy and photothermal therapy. The hydrophilic ICG was encapsulated into the core of liposomes, which were uptaked by NK cells. The NK cells recognize the targets and make the first-round killing to the tumor cells through secreting perforin and granzyme, and then via laser irradiation at wavelength $808 \mathrm{~nm}$, ICG can convert the light into heat to ablate the remaining tumor cells.

cancer PTT while the liposome can concurrently work as a drug delivery nanocarrier. Specifically, the synergistic therapy effect of ICG NPs coated with NK cells is carefully inspected to kill A549 cells, in which NK carrying ICG loaded liposomes can be used as a new theranostic agent to conduct both immunotherapy and PTT (Fig. 1). The findings of the present study demonstrate that the developed strategy can pave a new avenue for improved cancer theranostics.

\section{Materials and Methods}

\subsection{Preparation and characteristic of liposomal ICG nanoparticles}

Liposomal ICG nanoparticles (NPs) were prepared using lipid-film hydration and freeze-thaw cycles 
followed by extrusion method. 1, 2-dipalmitoyl-snglycero-3-phosphocholine (DPPC), 1, 2-distearoylsnglycero-3-phosphoethanolamine- $\mathrm{N}$-[methoxy(polyethyleneglycol)-2000] (ammonium salt) (DSPE-mPEG (2000), cholesterol (Avanti, USA), and ICG (Santa Cruz) were mixed in a tube, which was then attached to a vacuum rotary evaporator, allowing for the evaporation of organic solvents and formation of a lipid thin layer. The lipid film formed was hydrated and mixed with PBS, and then was freeze-thawed with 6 cycles freeze-thaw. The dispersion was then extruded 5 times through $100 \mathrm{~nm}$ polycarbonate membrane using Avanti Polar Lipids syringe extruder (Alabaster, AL, USA) to allow for the formation of liposomal structure. The hydrodynamic size (nm), polydispersity index and zeta potential $(\mathrm{mV})$ were determined by dynamic light scattering using a Zetasizer NanoZS instrument (Malvern, U.K.). And the UV-Vis absorbance of liposomal ICG was measured using an UV-Vis spectrophotometer (UV-1800, Shimadzu, Japan).

\subsection{Cell lines and cultures}

The human natural killer cells, NK-92MI, were purchased from the American Type Culture Collective (ATCC, US). And then they were cultured in the Alpha Minimum Essential medium ( $\alpha$-MEM) supplement with $12.5 \%$ horse serum and $12.5 \%$ fetal bovine serum (FBS). The cells were maintained at $37^{\circ} \mathrm{C}$ with $5 \% \mathrm{CO}_{2}$ in a humidified incubator.

\subsection{The photothermal properties of liposomal ICG NPs}

To access the photothermal properties of liposomal ICG NPs, $500 \mu \mathrm{l}$ of liposomal ICG solution with ICG concentrations at 2.5, 5, 10, 20 and $40 \mu \mathrm{g} / \mathrm{ml}$ was, respectively prepared by serial dilution using PBS. PBS was used as a blank control. The asprepared solution was respectively placed in $1.5 \mathrm{ml}$ eppendorf tubes and then irradiated by laser at wavelength $808 \mathrm{~nm}$ (power density $1 \mathrm{~W} / \mathrm{cm}^{2}$ ) for $8 \mathrm{~min}$. The temperature of each group was tracked every $10 \mathrm{~s}$ for a period of $8 \mathrm{~min}$ using an infrared thermal imaging camera (Ti27, Fluke, USA).

\subsection{In vitro cellular uptake of liposomal ICG by NK92MI cells}

NK92MI cells were cultured in a 96-well plate at the density of $5 \times 10^{4}$ cells/well and incubated with liposomal ICG solutions based on ICG concentrations of $3,15,30$ and $40 \mu \mathrm{g} / \mathrm{ml}$ for $4 \mathrm{~h}$ at $37^{\circ} \mathrm{C}$. NK92MI cells cultured without addition of liposomal ICG served as the control. After $4 \mathrm{~h}$ of incubation, the cells were harvested and washed three times with PBS and then quantitatively measured by flow cytometry (Accuri C6, BD, USA). To further analyze the most suitable incubation time for a better cellular uptake of liposomal ICG nanoparticle by NK92MI cells, cells were co-incubated with liposomal ICG $(40 \mu \mathrm{g} / \mathrm{ml})$ at $1,2,4$ and $6 \mathrm{~h}$ at $37^{\circ} \mathrm{C}$, respectively. The control group were cultured without addition of liposomal ICG. After incubation, the cells were harvested and washed three times with PBS and again measured by flow cytometry.

\subsection{Cell viability of liposomal ICG NPs}

The cytotoxicity of liposomal ICG to NK92MI cells was quantitatively measured through MTT (3-(4,5dimethylthiazol-2-yl)-2,5-diphenyltetrazolium bromide) assay (Sigma). NK-92MI cells were grown in $\alpha$-MEM medium and seeded in 96 -well plate at a density of $1 \times 104$ cells per well. The cells were then incubated with liposomal ICG at various ICG concentrations in triplicates and incubated for $4 \mathrm{~h}$. The untreated cells were used as the control. After $4 \mathrm{~h}$ incubation, the medium was replaced by fresh medium with $10 \mu \mathrm{l}$ of MTT solution $(5 \mu \mathrm{g} / \mathrm{ml})$ added to each well and further incubated for $4 \mathrm{~h}$ to allow for the formation of formazan crystals. After incubation, the plate was centrifuged at $1000 \times \mathrm{g}$ for $5 \mathrm{~min}$ and then aspirated the supernatant. $100 \mu \mathrm{l}$ of DMSO were then added to each well and further incubated for $30 \mathrm{~min}$ at $37^{\circ} \mathrm{C}$ to dissolve the formazan crystals. The absorbance was then quantified at $570 \mathrm{~nm}$ using multimode microplate reader (Spark 10 m, Tecan).

\subsection{Killing effect of NK cells carrying indocyanine green loaded liposomes to A549, Hela and MCF-7 cancer cells}

The cell mortality was measured using a calcein-AM release assay kit (Invitrogen, US). NK92MI cells were first incubated with liposomal ICG for $4 \mathrm{~h}$ to 


\section{S. Huang et al.}

complete the cellular uptake. Liposomal ICG NPs, after incubation, were washed with PBS for three times and resuspeneded in cell medium. Then, NK92MI cells loaded liposomal ICG NPs were added to A549, Hela cells and MCF-7 cells to mix each other with $3: 1,1: 1$ and 1:1 E:T ratio, respectively. After cell mixing, the plates were incubated at $37^{\circ} \mathrm{C}$ in $5 \% \quad \mathrm{CO}_{2}$ for $3 \mathrm{~h}$. The spontaneous release of calcein-AM was determined by incubating target cell in cell medium only and the maximum release of calcein -AM was determined by adding $1 \%$ Triton-X. After $3 \mathrm{~h}$ incubation, the laser irradiation group were irradiated with $808 \mathrm{~nm}$ laser at $1 \mathrm{~W} / \mathrm{cm}^{2}$ for $5 \mathrm{~min}$. Then, the plate was centrifuged at $300 \mathrm{~g}$ for $5 \mathrm{~min}$ and $100 \mu \mathrm{l} \mathrm{su}-$ pernatant were taken from each well and transferred to a 96-well white plate. The fluorescence of calcein -AM was measured by a multimode plate reader (EnVision 2105 Multimode Plate Reader, PerkinElmer) at 495/515 excitation/emission wavelength. The cell mortality was measured as the percentage of calcein release: [(experimental release-spontaneous release)/(maximum releasespontaneous release) $]^{*} 100 \%$.

\section{Results and Discussion}

\subsection{Preparation and characteristic of liposomal ICG}

The hydrodynamic size, polydispersity index and zeta potential were provided in Table 1 . The mean diameter of liposomal ICG was $108.1 \mathrm{~nm}$ (Fig. 2(a)) and the zeta potential was $-0.900 \mathrm{mV}$ (Fig. 2(b)) with polydispersity index of 0.072 , which exhibited the effect on nanoparticle size distribution. The UV absorption spectra of liposomal ICG was given in

Table 1. The general characteristics of liposomal ICG NPs.

\begin{tabular}{lccc}
\hline Character & $\begin{array}{c}\text { Average } \\
\text { diameter (nm) }\end{array}$ & $\begin{array}{c}\text { Polydispersity } \\
\text { index (PDI) }\end{array}$ & $\begin{array}{c}\text { Zeta } \\
\text { potential }(\mathrm{mV})\end{array}$ \\
\hline Liposomal ICG & $108.1 \mathrm{~nm}$ & 0.072 & $-0.900 \mathrm{mV}$ \\
\hline
\end{tabular}

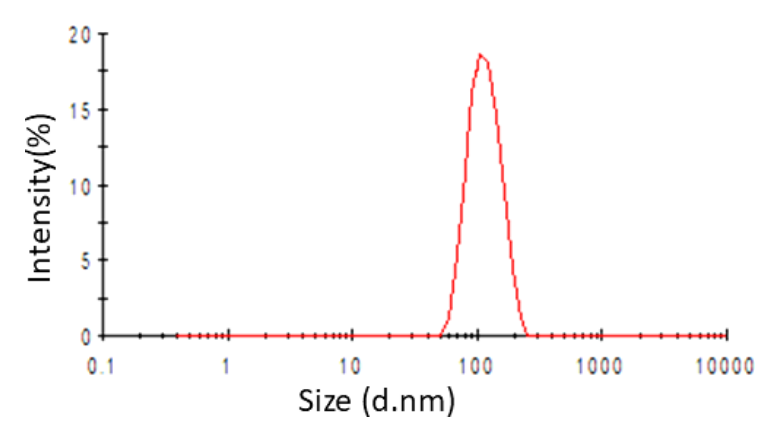

(a)

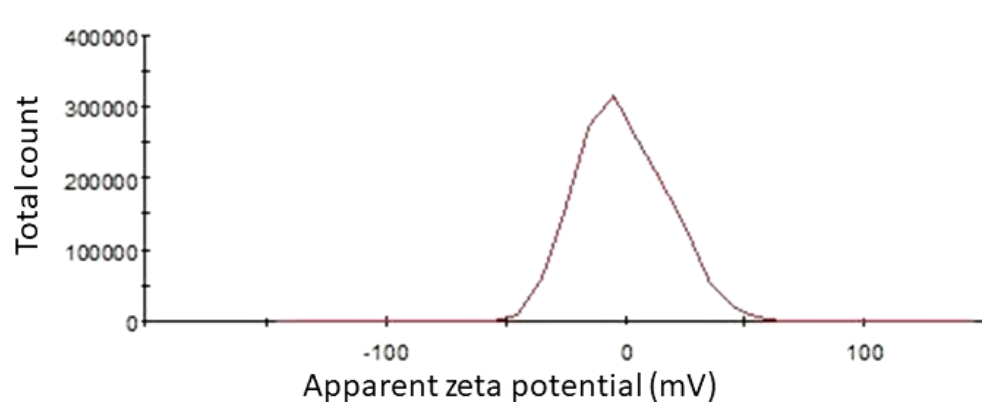

(b)

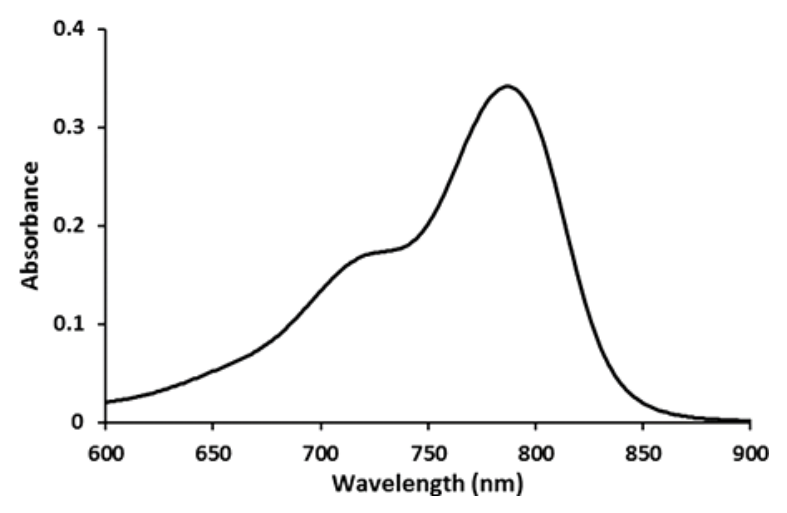

(c)

Fig. 2. Characterization of liposomal ICG micelles. (a) The size, (b) Zeta potential distribution of liposomal ICG by dynamic light scattering and (c) The UV-Vis absorption spectra of liposomal ICG. 
Fig. 2(c). Its absorbance peak was at $\lambda=794 \mathrm{~nm}$, which demonstrated that the developed theranostic probe was an excellent NIR light absorber for carrying out photothermal therapy.

\subsection{Evaluation of the photothermal properties of liposomal ICG NPs}

The photothermal properties of liposomal ICG NPs were evaluated under NIR laser irradiation for $8 \mathrm{~min}$ and the analysis results were illustrated in Fig. 3. It was discovered that upon NIR irradiation, the temperature of liposomal ICG solutions increased rapidly for the first $3 \mathrm{~min}$, and then gradually reached a plateau followed by slightly decreased temperature with increased irradiation time. For the control, PBS also received the same conditions of NIR irradiation but showed only a negligible temperature increase. The analysis results demonstrated that liposomal ICG is a suitable photothermal agent in carrying out photothermal therapy.

\subsection{In vitro cellular uptake of liposomal ICG by NK92MI cells}

The quantitative analysis results associated with cellular uptake by flow cytometry were illustrated in Fig. 4(a). Compared to the control group without incubating with liposomal ICG, stronger fluorescence signals were detected in the treated groups. The results illustrated that NK92MI cells incubated with the high concentration $(40 \mu \mathrm{g} / \mathrm{ml})$ of liposomal ICG exhibited significantly higher fluorescence intensity than the other groups (Fig. 4(b)). As a result, liposomal ICG NPs were able to be taken up by cells rapidly and efficiently when incubated with a high concentration. To further examine the best incubation time, liposomal ICG $(40 \mu \mathrm{g} / \mathrm{ml})$ were incubated with NK92MI cells with different time periods (1, 2, 4 and $6 \mathrm{~h}$ ) (Fig. 4(c)). We discovered that with a period of $4 \mathrm{~h}$ incubation, a slightly strong fluorescence signal was detected as compared to the other groups, indicating that $4 \mathrm{~h}$ is the most suitable period for incubation, allowing for a better cellular uptake (Fig. 4(d)). We have compared the ICG uptake of NK cells at $4^{\circ}$ and $37^{\circ}$ side by side. NK cells shows the improved efficiency at $37^{\circ}$ than $4^{\circ}$. (Supplementary information Fig. S1)

\subsection{Cell viability of liposomal ICG NPs}

The cytotoxicity of liposomal ICG to NK92MI cells was analyzed and the results were provided in Fig. 5(a). The cell viability of NK92MI exhibited no significant variation by uptaking liposomal ICG NPs, which indicated that the liposomal ICG NPs have better biocompatibility and show no toxicity to NK cells.

\subsection{Cytotoxicity of NK cells carrying indocyanine green loaded liposomes to different cancer cell lines}

The NK cells carrying indocyanine green loaded liposomes were measured to kill different cancer cell lines, including A549, Hela and MCF-7 cancer cells, as shown in Figs. 5(b), 5(c) and 5(d). We observed that cell mortality of NK cells carrying indocyanine green loaded liposomes irradiated by laser at wavelength $808 \mathrm{~nm}$ was $79.4 \%, 54.4 \%$ and $51.9 \%$, individually.

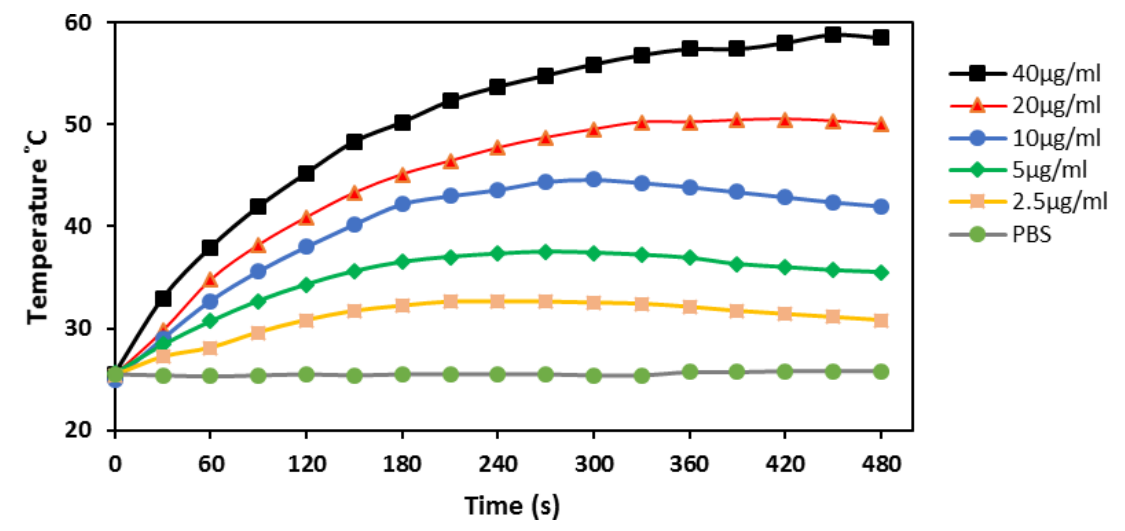

Fig. 3. The temperature profiles of liposomal ICG with different concentrations of ICG with laser irradiation at wavlenght $808 \mathrm{~nm}$ (power density $1 \mathrm{~W} / \mathrm{cm}^{2}$ ) for $8 \mathrm{~min}$. 


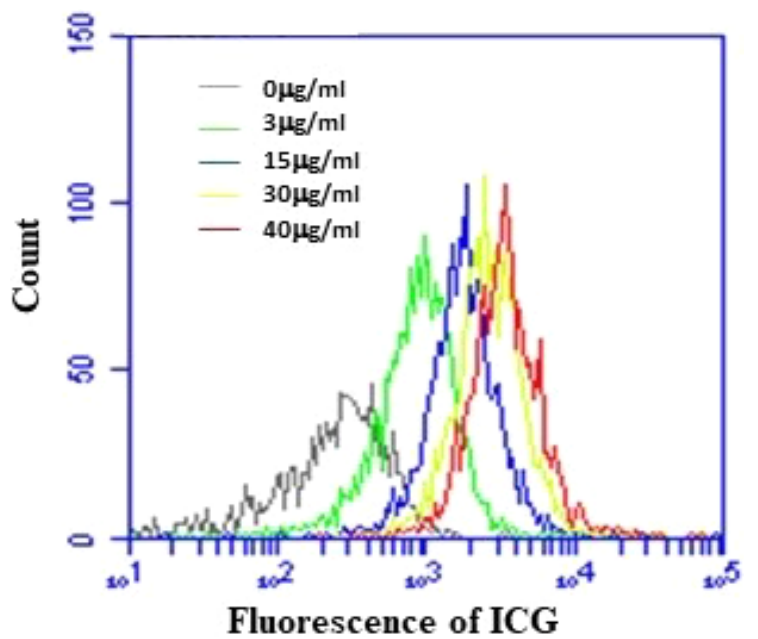

(a)

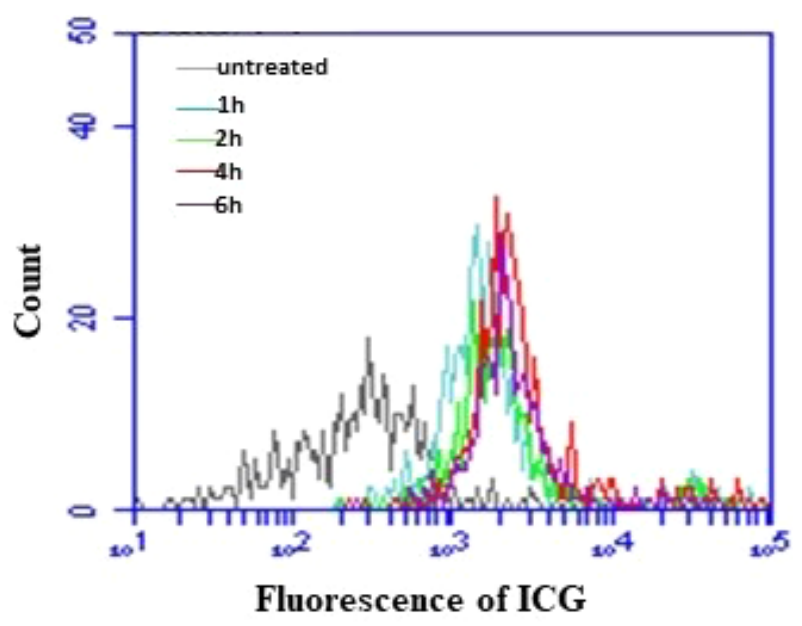

(c)

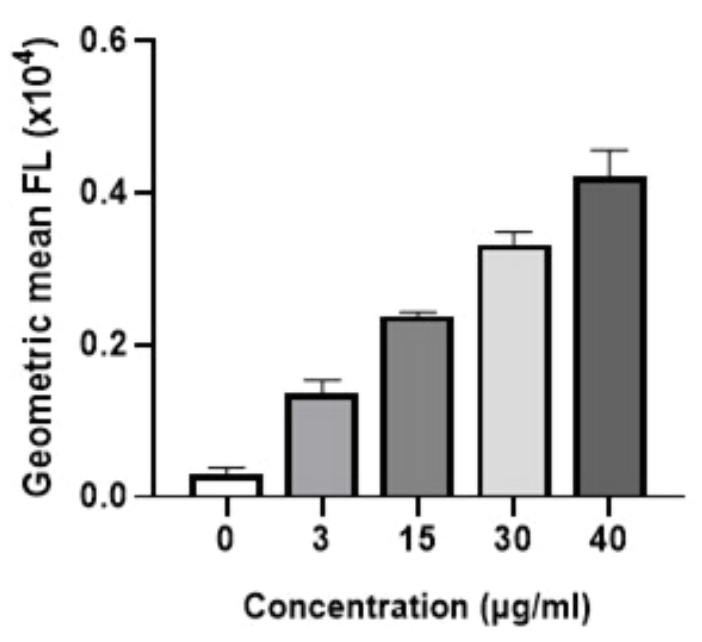

(b)

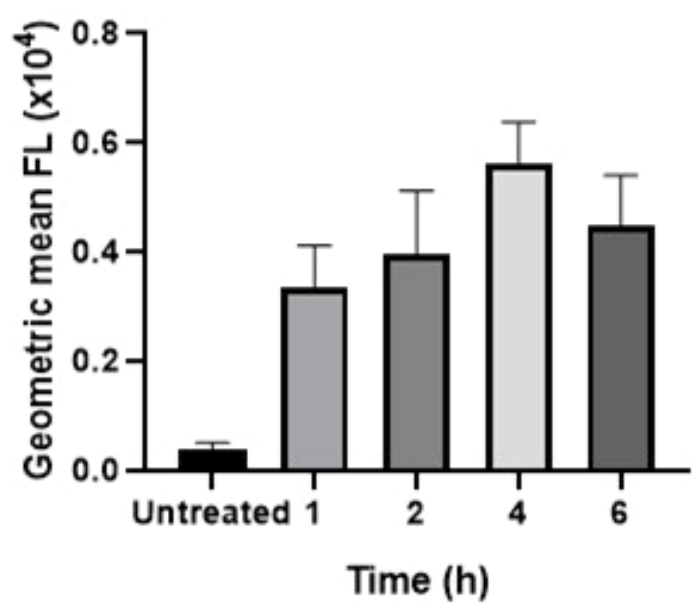

(d)

Fig. 4. Uptake analysis of NK92MI cells with liposomal ICG micelles. (a) The cellular uptake of NK92MI cells versus different concentrations of liposomal ICG was, respectively measured by flow cytometry, (b) The geometric mean fluorescence signals of NK92MI cells were summarized after incubated with different concentrations of liposomal ICG, (c) The cellular uptake of NK92MI cells with liposomal ICG during different time periods were measured by flow cytometry and (d) The geometric mean fluorescence signals of NK92MI cells were summarized after incubated with liposomal ICG during various time periods. The untreated group served as the control.

These results demonstrated that ICG-loaded NK cells had significantly improved anti-tumor cytotoxicity than the groups of sole ICG, NK vehicles, or the combination of NK vehicles and ICG.

\subsection{Discussion}

In this study, we synthesized liposomal ICG NPs, which can serve as the theranostic agent for concurrent immunotherapy and PPT. To the best of our knowledge, this is the first study that combines
NK cells with ICG NPs for enhanced cancer therapy. The analysis results demonstrated that the developed NK cells carrying indocyanine green loaded liposomes showed excellent capability and low cytotoxicity for killing cancer cells. However, in vivo animal tests should be performed in the future to examine the efficiency of the synergetic cancer treatment. In addition, a more precious immune cell such as CAR-NK should be adopted as well to coat NPs in order to conduct targeted cancer treatment. In particular, the therapy mechanism for synergetic 


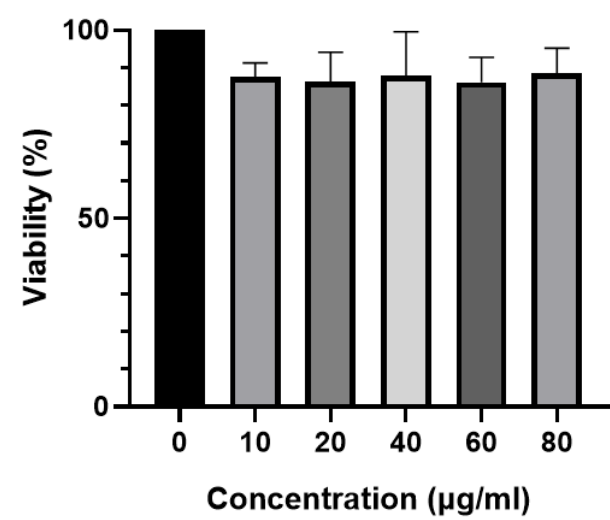

(a)

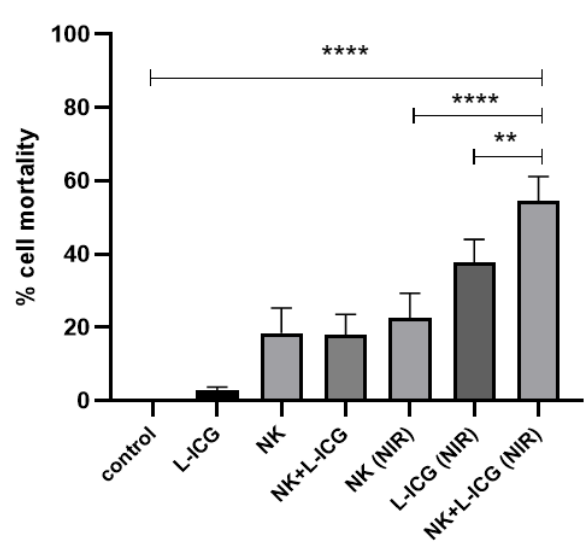

(c)

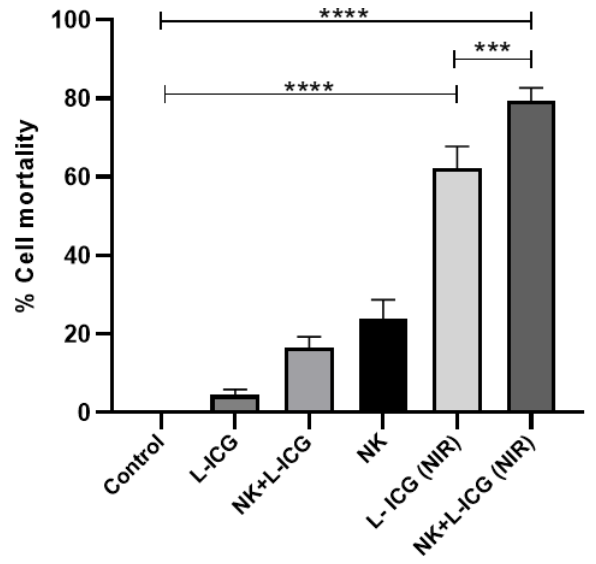

(b)

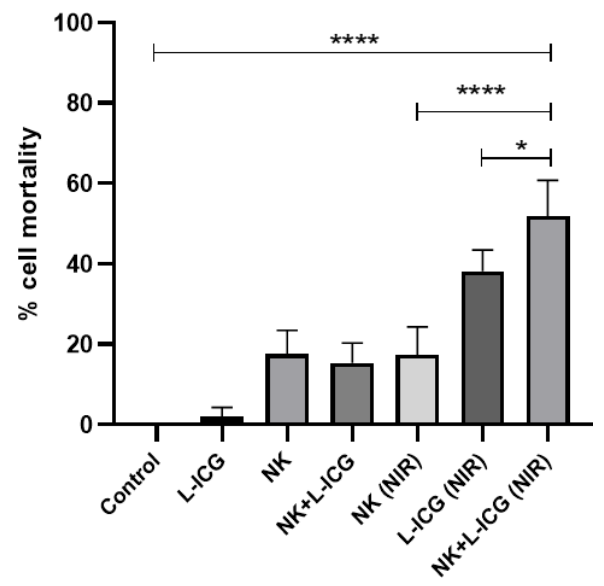

(d)

Fig. 5. Morphology of NK92MI cells loaded with nanoparticles. (a) Cell viability of NK92MI cells uptaked by liposomal ICG NPs with concentrations of 10, 20, 40,60 and $80 \mu \mathrm{g} / \mathrm{ml}$, respectively. Cell mortality of A549 cells, (b) Hela cells, (c) MCF-7 cells and (d) Treated by L-ICG, NK/L-ICG, NK, L-ICG(NIR) and NK/L-ICG(NIR), respectively.

treatment based on CAR-NK cells should be further explored to improve curative effect of cancer treatment.

\section{Conflict of Interest}

The authors of this work have nothing to disclose.

\section{Acknowledgments}

This work was supported by the Science and Technology Development Fund of Macau (FDCT/ 131/2016/A3, FDCT/0015/2018/A1, FDCT/ 0011/2018/A1), the Guangzhou Science and Technology Program (No. 201807010004), Start-up Research Grant (SRG2016-00082-FHS) and the intramural research program of Faculty of Health
Sciences, University of Macau, and National Natural Science Foundation of China (No. 31440041). First, second, third, fifth and sixth authors contributed equally to this work.

\section{References}

1. Y. Wang et al., "Small molecule inhibitors reveal allosteric regulation of USP14 via steric blockade," Cell Res. 28(12), 1186-1194 (2018).

2. Q. Zhao et al., "A dual-specific anti-IGF-1/IGF-2 human monoclonal antibody alone and in combination with temsirolimus for therapy of neuroblastoma," Int. J. Cancer 137(9), 2243-2252 (2015).

3. D. Z. Li et al., "N-terminal alpha-amino group modification of antibodies using a site-selective click chemistry method," MAbs 10(5), 712-719 (2018). 
4. D. Li et al., "Engineered antibody $\mathrm{CH} 2$ domains binding to nucleolin: Isolation, characterization and improvement of aggregation," Biochem. Biophys. Res. Commun. 485(2), 446-453 (2017).

5. Q. Zhao et al., "Human monoclonal antibody fragments binding to insulin-like growth factors I and II with picomolar affinity," Mol. Cancer Ther. 10(9), 1677-1685 (2011).

6. Q. Zhao, Z. Zhu, D. S. Dimitrov, "Yeast display of engineered antibody domains," Methods Mol. Biol. 899, 73-84 (2012).

7. Z. Chen et al., "Generation of bispecific antibodies by Fc heterodimerization and their application," Curr. Pharm. Biotechnol. 17, 1324-1332 (2016).

8. Z. Chen et al., "A dual-specific IGF-I/II human engineered antibody domain inhibits IGF signaling in breast cancer cells," Int. J. Biol. Sci. 14(7), 799806 (2018).

9. Y. Wang et al., "Screening and expressing HIV-1 specific antibody fragments in Saccharomyces cerevisiae," Mol. Immunol. 103, 279-285 (2018).

10. T. $\mathrm{Xu}$ et al., "A native-like bispecific antibody suppresses the inflammatory cytokine response by simultaneously neutralizing tumor necrosis factoralpha and interleukin-17A," Oncotarget 8(47), 81860-81872 (2017).

11. D. Li et al., "N-terminal residues of an HIV-1 gp41 membrane-proximal external region antigen influence broadly neutralizing 2F5-like antibodies," Virol. Sin. 30(6), 449-456 (2015).

12. Q. Zhao et al., "Alteration of electrostatic surface potential enhances affinity and tumor killing properties of anti-ganglioside GD2 monoclonal antibody hu3F8," J. Biol. Chem. 290(21), 13017-13027 (2015).

13. D. Chu et al., "Photosensitization priming of tumor microenvironments improves delivery of nanotherapeutics via neutrophil infiltration," Adv. Mater. 29 (27), (2017).

14. D. Chu et al., "Nanoparticle targeting of neutrophils for improved cancer immunotherapy," Adv. Health. Mater. 5(9), 1088-1093 (2016).

15. Y. Wu et al., "Recombinant-fully-human-antibody decorated highly-stable far-red AIEdots for in vivo HER-2 receptor-targeted imaging," Chem. Commun. (Camb) 54(53), 7314-7317 (2018).

16. Q. Zhao et al., "Affinity maturation of T-cell receptor-like antibodies for Wilms tumor 1 peptide greatly enhances therapeutic potential," Leukemia 29(11), 2238-2247 (2015).

17. D. L. Stanculeanu et al., "Development of new immunotherapy treatments in different cancer types," J. Med. Life 9(3), 240-248 (2016).
18. M. J. Smyth et al., "Activation of NK cell cytotoxicity," Mol. Immunol. 42(4), 501-510 (2005).

19. T. Tonn et al., "Treatment of patients with advanced cancer with the natural killer cell line NK-92," Cytotherapy 15(12), 1563-1570 (2013).

20. L. Boissel et al., "Retargeting NK-92 cells by means of CD19- and CD20-specific chimeric antigen receptors compares favorably with antibody-dependent cellular cytotoxicity," Oncoimmunol. 2(10), e26527 (2013).

21. H. Klingemann, L. Boissel, F. Toneguzzo, "Natural killer cells for immunotherapy - Advantages of the NK-92 cell line over blood NK cells," Front. Immunol. 7, 91 (2016).

22. Y. Chen et al., "Gene-modified NK-92MI cells expressing a chimeric CD16-BB-zeta or CD64-BBzeta receptor exhibit enhanced cancer-killing ability in combination with therapeutic antibody," Oncotarget 8(23), 37128-37139 (2017).

23. M. Ahmed et al., "Humanized affinity-matured monoclonal antibody $8 \mathrm{H} 9$ has potent antitumor activity and binds to FG loop of tumor antigen B7-H3," J. Biol. Chem. 290(50), 30018-30029 (2015).

24. N. J. Topham, E. W. Hewitt, "Natural killer cell cytotoxicity: How do they pull the trigger?," Immunology 128(1), 7-15 (2009).

25. L. Sercombe et al., "Advances and challenges of liposome assisted drug delivery," Front Pharmacol. 6, 286 (2015).

26. F. Xu et al., "Loading of indocyanine green within polydopamine-coated laponite nanodisks for targeted cancer photothermal and photodynamic therapy," Nanomater. (Basel) 8(5), 347 (2018).

27. J. R. Melamed, R. S. Edelstein, E. S. Day, "Elucidating the fundamental mechanisms of cell death triggered by photothermal therapy," $A C S$ Nano 9(1), 6-11 (2015).

28. N. Onda et al., "Preferential tumor cellular uptake and retention of indocyanine green for in vivo tumor imaging," Int. J. Cancer 139(3), 673-682 (2016).

29. S. Luo et al., "A review of NIR dyes in cancer targeting and imaging," Biomater. 32(29), 7127-7138 (2011).

30. H. J. Yoon et al., "Liposomal indocyanine green for enhanced photothermal therapy," ACS Appl. Mater. Interfaces 9(7), 5683-5691 (2017).

31. A. K. Kirchherr, A. Briel, K. Mader, "Stabilization of indocyanine green by encapsulation within micellar systems," Mol. Pharm. 6(2), 480-491 (2009). 